九州大学学術情報リポジトリ

Kyushu University Institutional Repository

\title{
Synthesis and Plant Growth-Inhibiting Activity of Pyridine derivatives
}

Tsukada, Hidetaka

Laboratory of Pesticide Chemistry, Faculty of Agriculture, Kyushu University

Kobayashi, Naoto

Laboratory of Pesticide Chemistry, Faculty of Agriculture, Kyushu University

Yamada, Naotaka

Laboratory of Pesticide Chemistry, Faculty of Agriculture, Kyushu University

Taniguchi, Eiji

Laboratory of Pesticide Chemistry, Faculty of Agriculture, Kyushu University

他

https://doi.org/10.5109/24263

出版情報：九州大学大学院農学研究院紀要. 43 (1/2)，pp. 169-180，1998-11. Kyushu University バージョン：

権利関係 : 
J. Fat: Agr., Kyushu Univ., 43 (1·2), 169-180 (1998)

\title{
Synthesis and Plant Growth-Inhibiting Activity of Pyridine derivatives
}

\author{
Hidetaka Tsukada, Naoto Kobayashi, Naotaka Yamada, \\ Eiji Taniguchi and Eiichi Kuwano
}

\author{
Laboratory of Pesticide Chemistry, Faculty of Agriculture, \\ Kyushu University, Fukuoka 812-8581, Japan \\ (Received June: 1, 1998 and accepted Argust 7, 1998)
}

\begin{abstract}
A number of 3-pyridyl-2-propenoic acid derivatives, pyridines possessing a $\gamma$-butyrolactone ring and related compounds were synthesized and evaluated for their activity by using a lettuce seedling test. Most of the pyridine derivatives inhibiled the growth of lettuce seedlings at concentrations ranging from 10 to $100 \mathrm{ppm}$. In the pyridine analogs of cinnamic acid, the 4-pyridine anlalog is more active than the 2 - and 3-isomers. Of the 3-(4-pyridyl)propenoic acid derivatives examined, the $N, N$-diethyl-3-(4-pyridyl)-2-propenamide $\mathbf{3 c - 3}$ was the most active, which caused more than $90 \%$ inhibition of the hypocotyl and root growth at $50 \mathrm{ppm}$. Pyridine analogs with a 1,4-bulanediol showed activity comparable to that of pyridyl $\gamma$-butyrolactone compounds, suggesting that the presence of the $\gamma$-butyrolactone was dispensable for the activity.
\end{abstract}

\section{INTRODUCTION}

In plants, a number of cytochrome P450 monooxygenases (P450s) involve in the biosynthesis of hormones, steroids, flavonoids, phytoalexins, lignans and lignin intermediates (Donaldson and Luster, 1991). Cinnamate 4-hydroxylase is a P450 which catalyzes the hydroxylation of trans-cinnamic acid into trans-p-coumaric acid, the first oxygenation step of the general phenylpropanoid metabolism in plants. This enzyme which is essential for plants has not been found in any invertebrate or vertebrate animals (Teutsch et al., 1993). It might be an unexploited target for development of a new herbicide.

On the other hand, P450s are known to be selectively inhibited by various imidazole, 1,2,4-triazole, pyridine, and pyrimidine derivatives. In these heterocyclic compounds, an $\mathrm{sp}^{2}$-nitrogen atom binds preferentially a heme iron atom of P450 active site to cause enzyme inactivation (Ortiz de Montellano and Reich, 1986). In view of the extraordinary potency of the pyridines as P450 inhibitors, we designed and synthesized a series of 3-pyridyl-2-propenoic acid derivatives, which is differing only in having a pyridine nucleus in place of a benzene ring of cinnamic acid, as an inhibitor of cinnamate 4-hydroxylase. Recently the total synthesis of novel lignans, haedoxans (Ishibashi and Taniguchi, 1989), and their derivatives (Yamauchi et al., 1992) have been accomplished via $\gamma$-butyrolactone derivatives as a key intermediate in our laboratories. Using this synthetic procedure, the pyridine derivatives possessing a $\gamma$-butyrolactone ring which have been not synthesized so far were designed in expectation of obtaining a new class of biological active compounds. In the present paper, we report the synthesis and plant 
growth-inhibiting activity of pyridine analogs of cinnamic acid and $\gamma$-butyrolactone.

\section{MATERIALS AND METHODS}

\section{Synthesis}

All melting points (mp) are uncorrected. ${ }^{1} \mathrm{H}-\mathrm{NMR}$ spectra were recorded on JNM-GX400 spectrometers with tetramethylsilane as an internal standard. Gravity column chromatography was carried out with Merck kiesegel $60 \mathrm{~F} 254(0.063-0.200 \mathrm{~mm}$, $70-230$ mesh ASTM) and Wakogel C-300 (45-75 $\mu \mathrm{m})$. TLC was performed on precoated $60 \mathrm{~F} 254$ silica gel plates $(0.25 \mathrm{~mm}, 0.5 \mathrm{~mm}$, or $1 \mathrm{~mm}$ thickness, $20 \times 20 \mathrm{~cm})$ supplied by $\mathrm{E}$. Merck.

Ethyl 3-(2-pyridyl)-2-propenoate (1a)

To a solution of diethyl ethoxycarbonylmethylphosphonate $(5.0 \mathrm{~g}, 18.7 \mathrm{mmol})$ and $\mathrm{K}_{2} \mathrm{CO}_{*}(5.2 \mathrm{~g}, 18.7 \mathrm{mmol})$ in water $(20 \mathrm{ml})$ was added with stirring 2-pyridinecarboxaldehyde $(2 \mathrm{~g}, 18.7 \mathrm{mmol})$ and the mixture was stirred for $12 \mathrm{hr}$ at room temperature. The product was extracted with ethyl acetate and the ethyl acetate solution was washed with water, brine, and dried over $\mathrm{Na}_{2} \mathrm{SO}_{1}$. After removal of the solvent, the residue was chromatographed on silica gel by elution with hexane-ethyl acetate ( $8: 1)$. Concentration of the early eluate under reduced pressure afforded $0.11 \mathrm{~g}(3.3 \%)$ of cis isomer (1a-2). NMR (CDCl: $) \delta: 1.26(3 \mathrm{H}, \mathrm{t}, J=7.3 \mathrm{~Hz}), 4.21(2 \mathrm{H}, \mathrm{q}, J=7.3 \mathrm{~Hz}), 6.14(1 \mathrm{H}, \mathrm{d}, J=12.7 \mathrm{~Hz})$, $6.95(1 \mathrm{H}, \mathrm{d} J=12.7 \mathrm{~Hz}), 7.18-7.26(1 \mathrm{H}, \mathrm{m}), 7.64-7.70(2 \mathrm{H}, \mathrm{m}), 8.59(1 \mathrm{H}, \mathrm{d}, J=4.9 \mathrm{~Hz})$. Trans isomer (1a-1) was eluted after 1a-2 with hexane-ethyl acetate (10:1) eluate, $3.1 \mathrm{~g}$ (94\%). NMR $\left(\mathrm{CDCl}_{3}\right) \delta: 1.34(3 \mathrm{H}, \mathrm{t}, J=7.3 \mathrm{~Hz}), 4.27(2 \mathrm{H}, \mathrm{q}, J=7.3 \mathrm{~Hz}), 6.92(1 \mathrm{H}, \mathrm{d}$, $J=15.9 \mathrm{~Hz}), 7.26(1 \mathrm{H}, \mathrm{d}, \mathrm{d}, J=7.8,4.9 \mathrm{~Hz}), 7.42(1 \mathrm{H}, \mathrm{d}, J=7.8 \mathrm{~Hz}), 7.65-7.73(1 \mathrm{H}, \mathrm{m}), 8.64$ $(1 \mathrm{H}, \mathrm{d}, J=3.9 \mathrm{~Hz})$.

Compounds $\mathbf{1 b}$ and $\mathbf{1 c}$ were prepared in the same manner as $\mathbf{1 a}$ with use of 3-and 4-pyridinecarboxaldehyde respectively, instead of 2-pyridine-carboxaldehyde.

Ethyl 3-(3-pyridyl)-2-propenoate (1b) Yield 51\%. NMR $\left(\mathrm{CDCl}_{3}\right) \delta: 1.35(3 \mathrm{H}, \mathrm{t}$, $J=7.0 \mathrm{~Hz}), 4.28(2 \mathrm{H}, \mathrm{q}, J=7.0 \mathrm{~Hz}), 6.52(1 \mathrm{H}, \mathrm{d}, J=16.1 \mathrm{~Hz}), 7.32(1 \mathrm{H}, \mathrm{d}, \mathrm{d}, J=8.0,4.7 \mathrm{~Hz})$, $7.67(1 \mathrm{H}, \mathrm{d}, J=16.1 \cdot \mathrm{Hz}), 7.84(1 \mathrm{H}, \mathrm{d}, J=8.0 \mathrm{~Hz}), 8.60(1 \mathrm{H}, \mathrm{d}, \mathrm{d}, J=4.7,2,0 \mathrm{~Hz}), 8.75(1 \mathrm{H}, \mathrm{d}$, $J=2.0 \mathrm{~Hz})$.

Ethyl $9-(4-p y r i d y l)-2-p r o p e n o a t e ~(1 e)$ Yield $59 \%$. Mp $64.5-65^{\circ} \mathrm{C}$. NMR $\left(\mathrm{CDCl}_{1}\right)$ $\delta: 1.35(3 \mathrm{H}, \mathrm{t}, J=7.3 \mathrm{~Hz}), 4.29(2 \mathrm{H}, \mathrm{q}, J=7.3 \mathrm{~Hz}), 6.59(1 \mathrm{H}, \mathrm{d}, J=16.1 \mathrm{~Hz}), 7.36(2 \mathrm{H}, \mathrm{d}$, $J=6.1 \mathrm{~Hz}), 7.59(1 \mathrm{H}, \mathrm{d}, J=16.1 \mathrm{~Hz}), 8.65(2 \mathrm{H}, \mathrm{d}, J=6.1 \mathrm{~Hz})$.

N-n-propyl-3-(4-pyridyl)-2-propenamide (3c-1)

A mixturc of $1 \mathrm{c}(1.0 \mathrm{~g}, 5.6 \mathrm{mmol})$ and $\mathrm{NaOH}(0.7 \mathrm{~g}, 5.6 \mathrm{mmol})$ in $8 \mathrm{ml}$ of water and 10 $\mathrm{ml}$ of ethanol was stirred for $24 \mathrm{hr}$ at room temperature. After removal of the solvent, the residue was acidified with acetic acid. The resulting precipitates were collected by filtration and recrystallized from dimethylformamide and water affording $0.57 \mathrm{~g} \mathrm{(68 \% )}$ of 3-(4-pyridyl)-2-propenoic acid (2c). To a solution of $\mathbf{2 c}(0.2 \mathrm{~g}, 1.34 \mathrm{mmol})$, 1-ethyl-3(3-dimethylaminopropyl) carbodiimide hydrochloride $(0.26 \mathrm{~g}, 1.34 \mathrm{mmol})$ and 1-hydroxy -benzotriazole $(0.18 \mathrm{~g}, 1.34 \mathrm{mmol})$ in $10 \mathrm{ml}$ of dichloromethane was added with stirring $n$-propylamine $(0.08 \mathrm{~g}, 1.34 \mathrm{mmol})$. After stirring for $24 \mathrm{hr}$ at room temperature, the dichloromethane solution was washed with $5 \% \mathrm{Na}_{2} \mathrm{CO}$ solution, water and brine, and dried over $\mathrm{Na}_{2} \mathrm{SO}_{4}$. After removal of the solvent, the residue was chromatographed on 
silica gel and eluted with hexane-ethyl acetate (1:2) to afford $70 \mathrm{mg}(28 \%)$ of $\mathbf{3 c - 1}$. NMR $\left(\mathrm{CDCl}_{3}\right) \delta: 0.98(3 \mathrm{H}, \mathrm{t}, J=7.3 \mathrm{~Hz}), 1.54-1.65(2 \mathrm{H}, \mathrm{m}), 3.37(2 \mathrm{H}, \mathrm{q}, J=6.8 \mathrm{~Hz}), 5.71(1 \mathrm{H}$, broad s), $6.54(1 \mathrm{H}, \mathrm{d}, J=15.6 \mathrm{~Hz}), 7.34(2 \mathrm{H}, \mathrm{d}, J=5.9 \mathrm{~Hz}), 7.55(1 \mathrm{H}, \mathrm{d}, J=15.6 \mathrm{~Hz}), 8.62$ $(2 \mathrm{H}, \mathrm{d}, J=5.9 \mathrm{~Hz})$.

Compounds $\mathbf{3 c}-\mathbf{2}$ and $\mathbf{3 c}-\mathbf{3}$ were prepared in the same manner as that for compound

$3 \mathbf{c}-\mathbf{1}$ from a corresponding amine, instead of $n$-propylamine.

$\mathrm{N-Hexyl-3-(4-pyridyl)-2-propenamide}$ (3c-2) Yield 44\%. NMR ( $\left.\mathrm{CDCl}_{3}\right) \delta: 0.89$ $(3 \mathrm{H}, \mathrm{t}, J=7.1 \mathrm{~Hz}), 1.23-1.40(6 \mathrm{H}, \mathrm{m}), 1.53-1.78(2 \mathrm{H}, \mathrm{m}), 3.39(2 \mathrm{H}, \mathrm{q}, J=6.7 \mathrm{~Hz}), 5.86(1 \mathrm{H}$, broad s), $6.56(1 \mathrm{H}, \mathrm{d}, J=15.6 \mathrm{~Hz}), 7.33(2 \mathrm{H}, \mathrm{d}, J=5.9 \mathrm{~Hz}), 7.54(1 \mathrm{H}, \mathrm{d}, J=15.6 \mathrm{~Hz}), 8.61$ $(2 \mathrm{H}, \mathrm{d}, J=5.9 \mathrm{~Hz})$.

N,N-Dielhyl-3-(4-pyridyl)-2-propenamide (3c-3) Yield 61\%. NMR (CDCl $) \delta$ : $1.20(3 \mathrm{H}, \mathrm{t}, J=7.1 \mathrm{~Hz}), 1.28(3 \mathrm{H}, \mathrm{t}, J=7.1 \mathrm{~Hz}), 3.48(2 \mathrm{H}, \mathrm{q}, J=7.1 \mathrm{~Hz}), 3.50(2 \mathrm{H}, \mathrm{q}$, $J=7.1 \mathrm{~Hz}), 7.00(1 \mathrm{H}, \mathrm{d}, J=15.4 \mathrm{~Hz}), 7.36(2 \mathrm{H}, \mathrm{d}, J=5.5 \mathrm{~Hz}), 7.60(1 \mathrm{H}, \mathrm{d}, J=15.4 \mathrm{~Hz}), 8.62$ $(2 \mathrm{H}, \mathrm{d}, J=5.5 \mathrm{~Hz})$.

3-(1-Hydroxy-1-pyridylmethyl)-4-vinyldihydro-2(3H)-furanones (5)

To a solution of diisopropylamine $(10 \mathrm{ml}, 0.14 \mathrm{~mol})$ in THF $(100 \mathrm{ml}$, distilled from $\left.\mathrm{LiAlH}_{4}\right)$ was added $n$-butyllithium $\left(87.5 \mathrm{ml}, 1.6 \mathrm{M}\right.$ solution in hexane) at $-78^{\circ} \mathrm{C}$ under nitrogen gas. After stirring for 15 minutes, a solution of $\beta$-vinyl- $\gamma$-butyrolactone $(4,10$ g, $0.05 \mathrm{~mol})$ in THF $(50 \mathrm{ml})$ was added dropwise, and then the mixture was stirred at $-78^{\circ} \mathrm{C}$ for 30 minutes. A solution of pyridinecarboxaldehyde $(11.7 \mathrm{~g}, 0.11 \mathrm{~mol})$ in $\mathrm{THF}$ $(50 \mathrm{ml})$ was added at $-78^{\circ} \mathrm{C}$. After stirring at $-78^{\circ} \mathrm{C}$ for $1 \mathrm{hr}$, to the mixture was added saturated aqueous $\mathrm{NH}_{4} \mathrm{Cl}$ solution $(50 \mathrm{~mL})$ and EtOAc $(1000 \mathrm{~mL})$. The organic layer was separated, washed with brine, and dried $\left(\mathrm{Na}_{2} \mathrm{SO}_{4}\right)$. Concentration followed by column chromatography (silicagel, EtOAc) gave aldols (erythro and threo). Recrystallizalion from $\mathrm{EtOAc}$ gave pure aldols $\mathbf{5}$.

2-Pyridylaldol (5a) Yield 47\%, white amorphous solid, mp 103-104 ${ }^{\circ} \mathrm{C}$. H-NMR (400 MHz, $\mathrm{CDCl}_{3}$, TMS) $\delta 3.02$ (d.d, $1 \mathrm{H}, J=2.93,9.76,-\mathrm{CH}-\mathrm{CH}(\mathrm{OH})-\mathrm{Ar}$ ), 3.27 (d.d.d, $1 \mathrm{H}$, $\left.J=8.79,8.79,8.79, \mathrm{CH}_{2}=\mathrm{CH}-\mathrm{CH}-\right), 3.88\left(\mathrm{t}, 1 \mathrm{H}, J=8.79,-\mathrm{C} I I_{2} \mathrm{O}-\right), 4.37(\mathrm{t}, 1 \mathrm{H}, J=8.79$, $\left.-\mathrm{CH}_{2} \mathrm{O}-\right), 4.56-4.62\left(\mathrm{~m}, 2 \mathrm{H}, \mathrm{CH}_{2}=\mathrm{CH}-\right), 5.40(\mathrm{~d}, 1 \mathrm{H}, J=4.39,-\mathrm{CH}(\mathrm{OH})-\mathrm{Ar}) 5.19-5.28(\mathrm{~m}$, $\left.1 \mathrm{H}, \mathrm{CH}_{2}=\mathrm{CH}-\right), 5.46(\mathrm{~s}, 1 \mathrm{H}, \mathrm{OH}), 7.20-7.23(\mathrm{~m}, 1 \mathrm{H}, \mathrm{Ar}-\mathrm{H}), 7.41-7.43(\mathrm{~m}, 1 \mathrm{H}, \mathrm{Ar}-H)$, 7.66-7.71 (m, 2H, Ar- $H)$, C-NMR $\left(100 \mathrm{MHz}, \mathrm{CDCl}_{3}\right)$ 177.34, 158.82, 148.08, 137.14, 135.70, 123.06, 121.03, 117.34, 70.81, 70.12, 52.67, 39.18, Anal. Found: C, 66.10; 11, 5.59; $\mathrm{N}, 6.33$. Calcd. for $\mathrm{C}_{12} \mathrm{H}_{13} \mathrm{NO}_{3}: \mathrm{C}, 65.74 ; \mathrm{H}, 5.98 ; \mathrm{N}, 6.39 \%$.

3-Pyridylaldol $(\mathbf{5 b})$ Yield $52 \%$, white amorphous solid, mp $114^{\circ} \mathrm{C}$. ${ }^{1} \mathrm{H}-\mathrm{NMR}(400$ $\mathrm{MHz}, \mathrm{CDCl}$, TMS) $\delta 2.80$ (d.d, $1 \mathrm{H}, J=2.93,9.28,-\mathrm{CH}-\mathrm{CH}(\mathrm{OH})-\mathrm{Ar}$ ), 3.41 (t, $1 \mathrm{H}, J=8.79$, $\left.\mathrm{CH}_{u}=\mathrm{CH} \mathrm{CH}\right), 3.89\left(\mathrm{t}, 1 \mathrm{H}, J=8.79, \mathrm{CH}_{2} \mathrm{O}\right), 4.39(\mathrm{t}, 1 \mathrm{H}, J=8.79, \mathrm{CH} \mathrm{O}), 4.704 .74(\mathrm{~m}$, $\left.2 \mathrm{H}, \mathrm{CH}_{2}=\mathrm{CH}-\right), 5.26-5.35\left(\mathrm{~m}, 1 \mathrm{H}, \mathrm{CH}_{2}=\mathrm{Cll}-\right), 5.48(\mathrm{~d}, 1 \mathrm{H}, J=1.95,-\mathrm{CH}(\mathrm{OH})-\mathrm{Ar}), 6.40$ (br.s, $1 \mathrm{H},-\mathrm{OH}), 7.24-7.34(\mathrm{~m}, 1 \mathrm{H}, \mathrm{Ar}-H), 7.77-7.80(\mathrm{~m}, 1 \mathrm{H}, \mathrm{Ar}-H), 8.36-8.41(\mathrm{~m}, 1 \mathrm{H}$, $\mathrm{Ar}-H), 8.49-8.54(\mathrm{~m}, 1 \mathrm{H}, \mathrm{Ar}-H),{ }^{13} \mathrm{C}-\mathrm{NMR}\left(100 \mathrm{MHz}, \mathrm{CDCl}_{3}\right)$ 176.98, 147.81, 135.57, $135.61,134.02,123.10,117.53,70.54,67.86,52.97,38.81$, Anal. Found: C, 65.96; H, 6.17; $\mathrm{N}, 6.29$. Calcd. for $\mathrm{C}_{12} \mathrm{H}_{13} \mathrm{NO}_{3}: \mathrm{C}, 65.74 ; \mathrm{H}, 5.98 ; \mathrm{N}, 6.39 \%$.

4-Pyridylaldol (5c) Yield 58\%, white amorphous solid, mp $130^{\circ} \mathrm{C} .{ }^{1} \mathrm{H}-\mathrm{NMR}(400$ $\mathrm{MHz}, \mathrm{CDCl}_{\mathrm{j}}$, TMS) $\delta 2.81-2.84(\mathrm{~m}, 1 \mathrm{H},-\mathrm{CH}-\mathrm{CH}(\mathrm{OH})-\mathrm{Ar}$ ), 3.32 (d.d.d, $1 \mathrm{H}, J=8.79,8.79$, $8.79, \mathrm{CH}_{2}=\mathrm{CH}-\mathrm{CH}-$ ), 3.65 (br.s, $1 \mathrm{H},-\mathrm{OH}$ ), 3.90 (d.d, $1 \mathrm{H}, J=9.27,9.27,-\mathrm{CH}_{2} \mathrm{O}-$ ), 4.39 (d.d, $\left.1 \mathrm{H}, J=8.79,8.79,-\mathrm{CH}_{2} \mathrm{O}-\right), 4.76-4.81\left(\mathrm{~m}, 2 \mathrm{H}, \mathrm{CH}_{2}=\mathrm{CH}-\right), 5.25-5.30\left(\mathrm{~m}, 1 \mathrm{H}, \mathrm{CH}_{2}=\mathrm{CH}-\right)$, 
$5.42(\mathrm{~s}, 1 \mathrm{H},-\mathrm{CH}(\mathrm{OH})-\mathrm{Ar}), 7.28-7.36(\mathrm{~m}, 2 \mathrm{H}, \mathrm{Ar}-H), 8.51-8.58(\mathrm{~m}, 2 \mathrm{H}, \mathrm{Ar}-\mathrm{H}),{ }^{13} \mathrm{C}-\mathrm{NMR}$ $\left(100 \mathrm{MHz}, \mathrm{CDCl}_{3}\right) 177.02,150.65,149.38,135.27,120.98,118.00,70.75,68.99,52.49$, 39.02, Anal. Found: $\mathrm{C}, 65.73 ; \mathrm{H}, 5.93 ; \mathrm{N}, 6.38$. Calcd. for $\mathrm{C}_{12} \mathrm{H}_{13} \mathrm{NO}_{3}$ : C, 65.74; $\mathrm{H}, 5.98 ; \mathrm{N}$, $6.39 \%$.

3-[1-(tert-Butyldimethylsilyl)oxy-1-pyridylmethyl]-4-vinyldihydro-2(3H)furanomes $(\mathbf{6})$

To a solution of $5(8.0 \mathrm{~g}, 0.04 \mathrm{~mol})$ and imidazole $(10.9 \mathrm{~g}, 0.16 \mathrm{~mol})$ in DMF $(200 \mathrm{ml})$ was added tert-butyldimeyhylsilyl chloride at room temperature. After stirring for 3 days, to the mixture was added water $(100 \mathrm{ml})$ and ether $(500 \mathrm{ml})$. The organic layer was sepa-rated, washed with water, brine, and dried $\left(\mathrm{Na}_{3} \mathrm{SO}_{4}\right)$. Concentration followed by columu chromatography (silicagel, EtOAc) gave pure silylaldol 6.

6 a Yield $82 \%$, colorless oil. ' $\mathrm{H}-\mathrm{NMR}\left(400 \mathrm{MHz}, \mathrm{CDCl}_{3}\right.$, TMS) $\delta-0.30\left(\mathrm{~s}, 3 \mathrm{H},-\mathrm{Si}_{-}-\mathrm{CH}_{3}\right.$ ), $-0.03\left(\mathrm{~s}, 3 \mathrm{H},-\mathrm{Si}-\mathrm{CH}_{3}\right), 0.79\left(\mathrm{~s}, 9 \mathrm{H},-\mathrm{Si}-\mathrm{CH}_{3}\right), 2.77$ (d.d, $1 \mathrm{H}, J=2.45,9.28$, $-\mathrm{CH}-\mathrm{CH}(\mathrm{OH})-\mathrm{Ar}), 3.30\left(\mathrm{t}, 1 \mathrm{H}, J=8.79, \mathrm{CH}_{3}=\mathrm{CH}-\mathrm{CH}-\right), 3.66-3.73\left(\mathrm{~m}, 1 \mathrm{H},-\mathrm{CH}_{2} \mathrm{O}-\right), 3.95$ $\left(\mathrm{t}, 1 \mathrm{H}, J=8.79,-\mathrm{CH} \mathrm{H}_{2} \mathrm{O}\right), 4.56\left(\mathrm{t}, 2 \mathrm{H}, J=10.25, \mathrm{CH}_{2}=\mathrm{CH}-\right), 5.69(\mathrm{~s}, 1 \mathrm{H},-\mathrm{CH}(\mathrm{OH})-\mathrm{Ar})$ 5.74-5.81 (m, $\left.1 \mathrm{H}, \mathrm{CH}_{2}=\mathrm{CH}-\right), 7.10-7.18(\mathrm{~m}, 1 \mathrm{H}, \mathrm{Ar}-H), 7.50-7.57(\mathrm{~m}, 2 \mathrm{H}, \mathrm{Ar}-H)$, 8.33-8.40 (m, 2H, $\mathrm{Ar}-\mathrm{H})$, ${ }^{13} \mathrm{C}-\mathrm{NMR}\left(100 \mathrm{MHz}, \mathrm{CDCl}_{3}\right)$ 177.81, 159.76, $147.42,136.89$, $134.58,122.79,120.72,119.44,69.49,52.58,41.33,25.90,18.12,-4.76,-6.10$, Anal. Found: $\mathrm{C}, 65.00 ; \mathrm{H}, 7.45 ; \mathrm{N}, 5.84$. Calcd. for $\mathrm{C}_{18} \mathrm{H}_{27} \mathrm{NO}_{3} \mathrm{Si}: \mathrm{C}, 64.83 ; \mathrm{H}, 8.16 ; \mathrm{N}, 4.20 \%$.

6 Yield $75 \%$, colorless oil. 'H-NMR (400 MHz, CDCl $\mathrm{M}_{3}$ TMS) $\delta 0.02\left(\mathrm{~s}, 3 \mathrm{H},-\mathrm{Si}-\mathrm{CH}_{3}\right.$ ), $0.23\left(\mathrm{~s}, 3 \mathrm{H},-\mathrm{Si}-\mathrm{CH}_{3}\right), 1.02\left(\mathrm{~s}, 9 \mathrm{H},-\mathrm{Si}-\mathrm{CH}_{3}\right), 3.03($ d.d, $1 \mathrm{H}, J=3.42,10.25$, $-\mathrm{CH}-\mathrm{CH}(\mathrm{OTBS})-\mathrm{Ar}), 3.12-3.16\left(\mathrm{~m}, \mathrm{lH}, \mathrm{CH}_{2}=\mathrm{CH}-\mathrm{CH}-\right), 3.92-4.01\left(\mathrm{~m}, 2 \mathrm{H},-\mathrm{CH}_{2} \mathrm{O}-\right.$ ), $4.11-4.20\left(\mathrm{~m}, 2 \mathrm{H},-\mathrm{CH}_{3} \mathrm{O}\right), 5.26-5.43\left(\mathrm{~m}, 2 \mathrm{H}, \mathrm{CH}_{2}=\mathrm{CH}-\right), 5.59(\mathrm{~d}, 1 \mathrm{H}, J=1.95$, -CH(OTBS)-Ar), 5.89-5.98 (m, 1H, $\left.\mathrm{CH}_{3}=\mathrm{CH}-\right), 7.32-7.38(\mathrm{~m}, 1 \mathrm{H}, \mathrm{Ar}-H), 7.62-7.87(\mathrm{~m}$, $1 \mathrm{H}, \mathrm{Ar}-H), 8.58-8.63(\mathrm{~m}, 1 \mathrm{H}, \mathrm{Ar}-H), 8.65-8.75(\mathrm{~m}, 1 \mathrm{H}, \mathrm{Ar}-H),{ }^{13} \mathrm{C}-\mathrm{NMR}(100 \mathrm{MHz}$, $\left.\mathrm{CDCl}_{3}\right) 176.32,147.30,136.07,135.72,134.92,123.01,117.36,70.78,70.38,53.67,41.47$, $25.65,-3.61,-4.89$, Anal. Found: $\mathrm{C}, 64.11 ; \mathrm{H}, 8.75 ; \mathrm{N}, 4.03$. Calcd. for $\mathrm{C}_{1 \mathrm{~s}} \mathrm{H}_{4 \mathrm{i}} \mathrm{NO}_{33} \mathrm{Si}: \mathrm{C}$, $64.83 ; \mathrm{H}, 8.16 ; \mathrm{N}, 4.20 \%$.

6c Yield $78 \%$, colorless oil. ${ }^{1} \mathrm{H}-\mathrm{NMR}\left(400 \mathrm{MHz}, \mathrm{CDCl}_{3}, \mathrm{TMS}\right) \delta-0.19\left(\mathrm{~s}, 3 \mathrm{H},-\mathrm{Si}-\mathrm{CH}_{3}\right)$, $0.04\left(\mathrm{~s}, 3 \mathrm{H},-\mathrm{Si}-\mathrm{CH}_{3}\right), 0.81\left(\mathrm{~s}, 9 \mathrm{H},-\mathrm{Si}-\mathrm{CH}_{3}\right), 2.75-2.81(\mathrm{~m}, 1 \mathrm{H},-\mathrm{CH}-\mathrm{CH}$ (OTBS)-Ar), $3.71-3.75\left(\mathrm{~m}, 1 \mathrm{H}, \mathrm{CH}_{2}=\mathrm{CH}-\mathrm{CH}-\right), 3.80\left(\mathrm{t}, 2 \mathrm{H}, J=7.82-8.78,-\mathrm{CH}_{2} \mathrm{O}-\right), 3.90-3.94(\mathrm{~m}, 2 \mathrm{H}$, $-\mathrm{CH}_{3} \mathrm{O}-$ ) $, 5.02-5.08\left(\mathrm{~m}, 2 \mathrm{H}, \mathrm{CH}_{2}=\mathrm{CH}-\right), 5.18$ (d, $1 \mathrm{H}, J=2.93,-\mathrm{CH}$ (OTBS)-Ar), 5.64-5.76 $\left(\mathrm{m}, 1 \mathrm{H}, \mathrm{CH}_{2}=\mathrm{CH}-\right), 7.32-7.38(\mathrm{~m}, 2 \mathrm{H}, \mathrm{Ar}-H), 8.40-8.51(\mathrm{~m}, 2 \mathrm{H}, \mathrm{Ar}-H),{ }^{13} \mathrm{C}-\mathrm{NMR}$ $\left(100 \mathrm{MHz}_{1} \mathrm{CDCl}_{3}\right)$ 174.86, 149.73, 135.76, 121.66, 120.86, 117.73, 70.91, 70.04, 53.61, 41.32, 25.61, 18.00, -4.92. Anal. Found: C, 63.31; H, 8.45; N, 4.15. Calcd. for $\mathrm{C}_{18} \mathrm{H}_{2 \mathrm{~T}} \mathrm{NO}_{3} \mathrm{Si}$ : C, $64.83 ; \mathrm{H}, 8.16 ; \mathrm{N}, 4.20 \%$.

3-[1-(lert-Butyldimethylsilyl)oxy-1-pyridylmethyl]-2-vinyl-1,4-butanediols (7)

To a solution of lithium aluminum hydride $(1.70 \mathrm{~g}, 45 \mathrm{mmol})$ in THF $(150 \mathrm{ml})$ was added $6(9.34 \mathrm{~g}, 30 \mathrm{mmol})$ in THF $(50 \mathrm{ml})$ at $-10^{\circ} \mathrm{C}$ under nitrogen gas. After stirring for $1 \mathrm{hr}$, to the reaction mixture was added saturated aqueous $\mathrm{MgSO}_{4}$ solution $(2 \mathrm{ml})$ and $\mathrm{K}_{4} \mathrm{CO}_{3}(8.29 \mathrm{~g}, 60 \mathrm{mmol})$. After stirring for $24 \mathrm{hr}$ at room temperature, the mixture was filtered through a celite pad, and the filtrate was concentrated by vacuo. Concentration followed by column chromatography (silicagel, EtOAc) gave pure diols $\mathbf{7}$.

2-Pyridyldiol (7a) Yicld 91\%, colorless oil. ' $\mathrm{H}-\mathrm{NMR}\left(400 \mathrm{MHz}, \mathrm{CDCl}_{3}\right.$, TMS) $\delta-0.30$ $\left(\mathrm{s}, 3 \mathrm{H},-\mathrm{Si}-\mathrm{CH}_{3}\right),-0.02\left(\mathrm{~s}, 3 \mathrm{H},-\mathrm{Si}-\mathrm{CH}_{3}\right), 0.80\left(\mathrm{~s}, 9 \mathrm{H},-\mathrm{Si}-\mathrm{CH}_{3}\right), 1.90-2.00(\mathrm{~m}, 1 \mathrm{H}$, 
$-\mathrm{CH}-\mathrm{CH}(\mathrm{OH})-\mathrm{Ar}), 2.49-2.56\left(\mathrm{~m}, 1 \mathrm{H}, \mathrm{CH}_{2}=\mathrm{CH}-\mathrm{CH}-\right), 3.14-3.17\left(\mathrm{~m}, 1 \mathrm{H},-\mathrm{CH}_{2} \mathrm{O}-\right)$, $3.64-3.74\left(\mathrm{~m}, 3 \mathrm{H},-\mathrm{CH}_{2} \mathrm{O}-\right), 4.97(\mathrm{~d}, 1 \mathrm{H}, J=7.32,-\mathrm{CH}(\mathrm{OH})-\mathrm{Ar}), 5.00-5.20(\mathrm{~m}, 2 \mathrm{H}$, $\left.\mathrm{CH}_{2}=\mathrm{CH}-\right)$ 5.78-5.92 (m, $\left.1 \mathrm{H}, \mathrm{CH}_{2}=\mathrm{CH}-\right), 7.19-7.24(\mathrm{~m}, 1 \mathrm{H}, \mathrm{Ar}-\mathrm{H}), 7.61-7.71(\mathrm{~m}, 1 \mathrm{H}$, $\mathrm{Ar}-H), 8.34-8.46(\mathrm{~m}, 2 \mathrm{H}, \mathrm{Ar}-H),{ }^{13} \mathrm{C}-\mathrm{NMR}\left(100 \mathrm{MHz}, \mathrm{CDCl}_{3}\right) 162.72,148.01,13.23$, $137.22,122.65,121.75,116.25,75.12,62.08,60.38,58.40,51.62,43.68,25.76,14.16,-4.58$. Anal. Found: $\mathrm{C}, 63.37 ; \mathrm{H}, 9.22 ; \mathrm{N}, 4.00$. Calcd. for $\mathrm{C}_{15} \mathrm{H}_{31} \mathrm{NO}_{; 3} \mathrm{Si}: \mathrm{C}, 64.05 ; \mathrm{H}, 9.26 ; \mathrm{N}$, $4.15 \%$.

3-Pyridyldiol (7b) Yield 92\%, colorless oil. 'H-NMR (400 MHz, CDCl, 'TMS) $\delta-0.03$ $\left(\mathrm{s}, 3 \mathrm{H},-\mathrm{Si}-\mathrm{CH}_{3}\right),-1.02\left(\mathrm{~s}, 3 \mathrm{H},-\mathrm{Si}-\mathrm{CH}_{3}\right), 0.80\left(\mathrm{~s}, 9 \mathrm{H},-\mathrm{Si}-\mathrm{CH}_{3}\right), 1.90-2.00(\mathrm{~m}, 1 \mathrm{H}$, $-\mathrm{CH}-\mathrm{CH}(\mathrm{OTBS})-\mathrm{Ar}), 2.49-2.56\left(\mathrm{~m}, 1 \mathrm{H}, \mathrm{CH}_{2}=\mathrm{CH}-\mathrm{CH}-\right), 3.14-3.17\left(\mathrm{~m}, 1 \mathrm{H},-\mathrm{CH}_{2} \mathrm{O}-\right)$, $3.64-3.74\left(\mathrm{~m}, 3 \mathrm{H},-\mathrm{C} \mathrm{H}_{2} \mathrm{O}-\right), 4.97(\mathrm{~d}, 1 \mathrm{H}, J=7.32,-\mathrm{CH}$ (OTBS)-Ar), 5.00-5.20 (m, $2 \mathrm{H}$, $\mathrm{CH}=\mathrm{CH}-), 5.78-5.92\left(\mathrm{~m}, 1 \mathrm{H}, \mathrm{CH}_{2}=\mathrm{CH}-\right), 7.19-7.24(\mathrm{~m}, 1 \mathrm{H}, \mathrm{Ar}-H), 7.61-7.71(\mathrm{~m}, 1 \mathrm{H}$, $\mathrm{Ar}-H), 8.34-8.46(\mathrm{~m}, 2 \mathrm{H}, \mathrm{Ar}-H),{ }^{12} \mathrm{C}-\mathrm{NMR}\left(100 \mathrm{MHz}, \mathrm{CDCl}_{4}\right) 148.43,148.20,147.81$, $139.58,135.27,123.58,116.76,71.97,62.33,58.62,51.85,43.97,25.92,18.19,-4.27,-4.95$. Anal. Found: C, 63.90; H, 9.27; N, 3.95. Caled. for $\mathrm{C}_{15} \mathrm{H}_{31} \mathrm{NO}_{3} \mathrm{Si}$ : C, 64.05; H, 9.26; N, $4.15 \%$.

4-Pyridyldiol (7e) Yield 96\%, colorless oil. 'H-NMR (400 MHz, $\left.\mathrm{CDCl}_{3}, \mathrm{TMS}\right) \hat{\delta}-0.27$ $\left(\mathrm{s}, 3 \mathrm{H},-\mathrm{Si}-\mathrm{CH}_{3}\right),-0.01\left(\mathrm{~s}, 3 \mathrm{H},-\mathrm{Si}-\mathrm{CH}_{3}\right), 0.82\left(\mathrm{~s}, 9 \mathrm{H},-\mathrm{Si}-\mathrm{CH}_{3}\right), 1.89-1.95(\mathrm{~m}, 1 \mathrm{H}$, $-\mathrm{CH}-\mathrm{CH}(\mathrm{OTBS})-\mathrm{Ar}), 2.39-2.43\left(\mathrm{~m}, 1 \mathrm{H}, \mathrm{CH}_{2}=\mathrm{CH}-\mathrm{CH}-\right.$ ), 3.30 (d.d, $2 \mathrm{H}, J=5.37,11.23$, $-\mathrm{CH}_{2} \mathrm{O}-$ ) $3.57-3.66\left(\mathrm{~m}, 2 \mathrm{H},-\mathrm{CH}_{2} \mathrm{O}-\right), 4.94$ (d, $1 \mathrm{H}, J=5.86,-\mathrm{CH}$ (OTBS)-Ar), $5.01(\mathrm{~m}, 2 \mathrm{H}$, $\left.\mathrm{CH}_{2}=\mathrm{CH}-\right), 5.75-5.84\left(\mathrm{~m}, 1 \mathrm{H}, \mathrm{CH}_{2}=\mathrm{CH}-\right), 7.23-7.28(\mathrm{~m}, 2 \mathrm{H}, \mathrm{Ar}-H), 8.39-8.48(\mathrm{~m}, 2 \mathrm{H}$; $\mathrm{Ar}-H)$, ${ }^{\mathrm{C}} \mathrm{C}-\mathrm{NMR}\left(100 \mathrm{MHz}, \mathrm{CDCl}_{3}\right)$ 152.21, 149.34, 122.04, 122.03, 117.00, 72.91, 62.47, $59.16,51.17,43.69,25.75,18.07,-4.59,-4.40$. Anal. Found: C, $63.37 ; \mathrm{H}, 9.22 ; \mathrm{N}, 4.00$. Calcd. for $\mathrm{C}_{1 \times} \mathrm{H}_{31} \mathrm{NO}_{3} \mathrm{Si}: \mathrm{C}, 64.05 ; \mathrm{H}, 9.26 ; \mathrm{N}, 4.15 \%$.

3-11-(tert-Butyldimethylsilyl)oxy-1-pyridylmethyl]-3-hydroxymethyl-2hydroxydihydro-2(3H)-furanols (8)

To a stirred solution of $7(5 \mathrm{~g}, 15 \mathrm{mmol})$ and $N$-methylmorpholine $N$-oxide $(3.5 \mathrm{~g}, 30$ mmol) in acetone: $t$-butanol: $\mathrm{H}_{3} \mathrm{O}=4: 1: 1(150 \mathrm{ml})$ was added osmium tetraoxide $(0.5 \mathrm{ml}$, $2 \%$ in water) under nitrogen gas. After stirring for 3 days, the reaction mixture was quenched by saturated aqueous $\mathrm{Na}_{2} \mathrm{SO}_{3}$ solution $(5 \mathrm{ml})$. $A$ fter stirring for 30 minutes, the mixture was filtered through a celite pad, and the filtrate was concentrated by vacuo. The residue was dissolved in EtOAc $(200 \mathrm{mi})$, and to the mixture was added $\mathrm{NaIO}_{4}(3.9 \mathrm{~g}$, $180 \mathrm{mmol}$ ) in water $(50 \mathrm{ml})$. After vigorously stirring for $24 \mathrm{hr}$, the reaction mixture was diluted with EtOAc $(50 \mathrm{ml})$. The organic layer was separated, washed with water, brine, and dried $\left(\mathrm{Na}_{3} \mathrm{SO}_{4}\right)$. Concentration followed by column chromatography (silicagel, EtOAc) gave pure lactols $\mathbf{8}$.

2-Pyridyllactol (8a) Yield 91\%, colorless oil. ${ }^{1} \mathrm{H}-\mathrm{NMR}\left(400 \mathrm{MHz}, \mathrm{CDCl}_{3}, \mathrm{TMS}\right) \delta$ $-0.37\left(\mathrm{~s}, 3 \mathrm{H},-\mathrm{Si}-\mathrm{CH}_{i}\right),-0.03\left(\mathrm{~s}, 3 \mathrm{H},-\mathrm{Si}-\mathrm{CH}_{3}\right), 0.82\left(\mathrm{~s}, 9 \mathrm{H},-\mathrm{Si}-\mathrm{CH}_{3}\right), 2.27-2.29(\mathrm{~m}, 1 \mathrm{H}$, $-\mathrm{CH}-\mathrm{CH}(\mathrm{OH})-\mathrm{Ar}$ ), 2.41 (br.s, $1 \mathrm{H},-\mathrm{O} / \mathrm{f}), 3.07-3.11\left(\mathrm{~m}, 1 \mathrm{H}, \mathrm{HO}-\mathrm{CH}_{2}-\mathrm{CH}-\right.$ ) , 3.61-3.63 (m, $\left.4 \mathrm{H}, \mathrm{HO}-\mathrm{CH}_{\mathrm{L}},-\mathrm{CH}_{2} \mathrm{O}-\right), 3.85(\mathrm{~d}, 1 \mathrm{H}, J=5.37,11.23,-\mathrm{CH}(\mathrm{OH})-), 4.83(\mathrm{~d}, 1 \mathrm{H}, J-7.33$, -CH(OTBS)-Ar), 5.38 (br.s, $1 \mathrm{H},-\mathrm{OH}), 7.12-7.15(\mathrm{~m}, 1 \mathrm{H}, \mathrm{Ar}-H), 7.40-7.43(\mathrm{~m}, 1 \mathrm{H}$, $\mathrm{Ar}-H), 7.63-7.67(\mathrm{~m}, 1 \mathrm{H}, \mathrm{Ar}-\mathrm{H}), 8.39-8.43(\mathrm{~m}, 1 \mathrm{H}, \mathrm{Ar}-H) .{ }^{13} \mathrm{C}-\mathrm{NMR}(100 \mathrm{MHz}, \mathrm{CDCl})$ $162.40,148.21,137.10,122.80,121.25,101.10,74.14,68.23,59.49,49.61,46.82,25.73$, $18.00,-4.52,-4.74$. Anal. Found: $\mathrm{C}, 61.27 ; \mathrm{H}, 8.45 ; \mathrm{N}, 3.97$. Caled. for $\mathrm{C}_{17} \mathrm{H}_{27} \mathrm{NO}_{3} \mathrm{Si}$ : C, $60.14 ; \mathrm{H}, 8.61 ; \mathrm{N}, 4.13 \%$. 
3-Pyridyllactol (8b) Yield $63 \%$, white amorphous solid, $\mathrm{mp} 116{ }^{\circ} \mathrm{C} .{ }^{1} \mathrm{H}-\mathrm{NMR}$ $\left(400 \mathrm{MHz}, \mathrm{CDCl}_{3}, \mathrm{TMS}\right) \delta-6.64\left(\mathrm{~s}, 3 \mathrm{H},-\mathrm{Si}-\mathrm{CH}_{3}\right),-0.03\left(\mathrm{~s}, 3 \mathrm{H},-\mathrm{Si}-\mathrm{CH}_{3}\right), 0.80(\mathrm{~s}, 9 \mathrm{H}$, $-\mathrm{Si}-\mathrm{CH}_{3}$ ), 1.74 (br.s, $\left.1 \mathrm{H},-\mathrm{OH}\right), 2.33-2.38$ (m, $1 \mathrm{H},-\mathrm{CH}-\mathrm{CH}(\mathrm{OTBS})-\mathrm{Ar}$ ), 3.06-3.12 (m, 1H, $\mathrm{HO}-\mathrm{CH}_{3}-\mathrm{CH}-$-), 3.42 (d.d, $2 \mathrm{H}, J=8.79,8.79,-\mathrm{CH}_{2} \mathrm{O}-$ ), 3.63 (d.d, $2 \mathrm{H}, J=8.31,8.31$, $\mathrm{HO}-\mathrm{C} I_{:-}$), 3.89 (d.d, $1 \mathrm{H}, J=4.2,11.04,-\mathrm{CH}(\mathrm{OH})-$ ), 4.67 (d, $1 \mathrm{H}, J=8.79$, -CH(OTBS)-Ar), $5.47\left(\mathrm{~s}, 1 \mathrm{H}, H \mathrm{O}-\mathrm{CH}_{3}-\right), 7.22-7.25(\mathrm{~m}, 1 \mathrm{H}, \mathrm{Ar}-\mathrm{H}), 7.62-7.77(\mathrm{~m}, 1 \mathrm{H}$, $\mathrm{Ar}-H), 8.44-8.52(\mathrm{~m}, 2 \mathrm{H}, \mathrm{Ar}-H),{ }^{13} \mathrm{C}-\mathrm{NMR}\left(100 \mathrm{MHz}, \mathrm{CDCl}_{3}\right)$ 149.40, 147.94, 139.42, $134.81,124.00,101.73,71.55,68.94,59.88,49.46,47.72,26.05,25.83,18.17,-3.78,-4.95$. Anal. Found: $\mathrm{C}, 59.88 ; \mathrm{H}, 8.45 ; \mathrm{N}, 4.08$. Calcd. for $\mathrm{C}_{47} \mathrm{H}_{37} \mathrm{NO}_{33} \mathrm{Si}$ : C, 60.14; $\mathrm{H}, 8.61 ; \mathrm{N}, 4.13 \%$.

4-Pyridyllactol (8c) Yicld 53\%, white needle solid, mp $137-138^{\circ} \mathrm{C}$. 'H-NMR (400 $\mathrm{MHz}, \mathrm{CDCl}, \mathrm{TMS}) \delta-6.64\left(\mathrm{~s}, 3 \mathrm{H},-\mathrm{Si}-\mathrm{CH}_{3}\right),-0.03\left(\mathrm{~s}, 3 \mathrm{H},-\mathrm{Si}-\mathrm{CH}_{3}\right), 0.80\left(\mathrm{~s}, 9 \mathrm{H},-\mathrm{Si}-\mathrm{CH}_{3}\right)$, $2.27-2.31(\mathrm{~m}, \quad 1 \mathrm{H}, \quad-\mathrm{CH}-\mathrm{CH}(\mathrm{O} T \mathrm{TS})-\mathrm{Ar}), \quad 2.41-2.43 \quad\left(\mathrm{~m}, \quad 1 \mathrm{H}, \quad \mathrm{HO}-\mathrm{CH}_{2}-\right.$ $\mathrm{CH}-,), 3.33-3.67\left(\mathrm{~m}, 4 \mathrm{H},-\mathrm{CH}_{2} \mathrm{O}-, \mathrm{HO}-\mathrm{C} H_{-}-\right), 3.83-3.89(\mathrm{~m}, 1 \mathrm{H},-\mathrm{CH}(\mathrm{OH})-), 4.66(\mathrm{~d}, 1 \mathrm{H}$, $J=8.40,-\mathrm{CH}(\mathrm{OTBS})-\mathrm{Ar}), 5.43\left(\mathrm{~s}, 1 \mathrm{H}, \mathrm{HO}-\mathrm{CH}_{2}-\right), 7.19-7.20(\mathrm{~m}, 2 \mathrm{H}, \mathrm{Ar}-\mathrm{H}), 8.48-8.50(\mathrm{~m}$, $2 \mathrm{H}, \mathrm{Ar}-\mathrm{H}),{ }^{13} \mathrm{C}-\mathrm{NMR}\left(100 \mathrm{MHz}, \mathrm{CDCl}_{3}\right)$ 152.49, 149.49, 149.82, 121.58, 121.38, 101.65, $72.37,68.82,60.04,49.05,47.02,25.68,17.98,-4.12,-3.95$. Anal. Found: C, 59.77; H, $8.45 ; \mathrm{N}, 4.07$. Calcd. for $\mathrm{C}_{17} \mathrm{H}_{27} \mathrm{NO}_{3} \mathrm{Si}: \mathrm{C}, 60.14 ; \mathrm{H}, 8.61 ; \mathrm{N}, 4.13 \%$.

4-I1-(tert-Butyldimethylsilyl)oxy-1-pyridylmethyl]-3-hydroxymethyl-2-dihydro2(3H)-furanones $(\mathbf{9})$

A vigorously stirred solution of $\mathbf{8}(1.74 \mathrm{~g}, 5.13 \mathrm{~mm} n \mathrm{l})$ and $\mathrm{Ag}_{2} \mathrm{CO}_{3}$ celite $(4.62 \mathrm{~g}, 1$ mmol $/ 0.6 \mathrm{~g}, 7.7 \mathrm{mmol}$ ) in benzene $(50 \mathrm{ml})$ was heated under refluxing for 30 minutes. The reaction mixture was filtered through a celite pad. Concentration followed by column chromatography (silicagel, EtOAc) gave pure lactones $\mathbf{9}$.

2-Pyridyllactone (9a) Yield 54\%, white amorphous solid, mp $62-63^{\circ} \mathrm{C}$. 'H-NMR $\left(400 \mathrm{MHz}, \mathrm{CDCl}_{3}, \mathrm{TMS}\right) \delta-0.27\left(\mathrm{~s}, 3 \mathrm{H},-\mathrm{Si}_{-}-\mathrm{CH}_{3}\right),-0.03\left(\mathrm{~s}, 3 \mathrm{H},-\mathrm{Si}-\mathrm{CH}_{3}\right), 0.81(\mathrm{~s}, 9 \mathrm{H}$, $\left.-\mathrm{Si}-\mathrm{CH}_{3}\right), 2.65-2.70(\mathrm{~m}, 1 \mathrm{H},-\mathrm{CH}-\mathrm{CH}(\mathrm{OH})-\mathrm{Ar}), 3.12-3.19\left(\mathrm{~m}, 1 \mathrm{H}, \mathrm{HO}-\mathrm{CH}_{2}-\mathrm{CH}-\right)$, 3.73-3.74 (m, 2H, $\left.\mathrm{HO}-\mathrm{CH}_{2}-,-\mathrm{CH}_{3} \mathrm{O}-\right), 3.97-4.04\left(\mathrm{~m}, 2 \mathrm{H}, \mathrm{HO}-\mathrm{CH}_{2}-,-\mathrm{CH}_{2} \mathrm{O}-\right.$ ), 4.01 (d.d, $1 \mathrm{H}, J=5.37,4.40, \mathrm{HO}-\mathrm{CH}-), 5.02(\mathrm{~d}, 1 \mathrm{H}, J=4.40,-\mathrm{CH}(\mathrm{OTBS})-\mathrm{Ar}), 7.05-7.36(\mathrm{mt}, 1 \mathrm{H}$, $\mathrm{Ar}-H), 7.41-7.56(\mathrm{~m}, 1 \mathrm{H}, \mathrm{Ar}-H), 7.57-7.59(\mathrm{~m}, 1 \mathrm{H}, \Lambda \mathrm{r}-I I), 8.34-8.43(\mathrm{~m}, 1 \mathrm{H}, \mathrm{Ar}-I H) . \quad \mathrm{C}$ -NMR (100 MHz, CDCl:) 178.45) 161.16, 148.96, 140.13, 137.39, 123.21, 121.14, 75.06, $69.99,60.69,59.72,45.33,44.91,26.05,18.30,-4.31,-4.82$. Anal. Found: C, 59.97; H, $7.91 ; \mathrm{N}, 4.07$. Calcd. for $\mathrm{C}_{17} \mathrm{H}_{2 ;} \mathrm{NO}_{4} \mathrm{Si}: \mathrm{C}, 60.5 ; \mathrm{H}, 8.06 ; \mathrm{N}, 4.15 \%$.

3-Pyridyllactone (9b) Yield $99 \%$, white amorphous solid, mp 94-95 $\mathrm{C}$. 'H-NMR $\left(400 \mathrm{MHz}, \mathrm{CDCl}_{3}, \mathrm{TMS}\right) \delta-0.03\left(\mathrm{~s}, 3 \mathrm{H},-\mathrm{Si}-\mathrm{CH}_{3}\right),-0.49\left(\mathrm{~s}, 3 \mathrm{H},-\mathrm{Si}-\mathrm{CH}_{3}\right), 0.80(\mathrm{~s}, 9 \mathrm{H}$, $\left.-\mathrm{Si}-\mathrm{CH}_{3}\right), 3.17-3.21(\mathrm{~m}, 1 \mathrm{H},-\mathrm{CH}-\mathrm{CH}(\mathrm{OTBS})-\mathrm{Ar}), 3.64-3.65\left(\mathrm{~m}, 1 \mathrm{H}, \mathrm{HO}-\mathrm{CH}_{-}-\mathrm{CH}-,\right), 4.10$ (d.d, $2 \mathrm{H}, J=8.91,8.91,-\mathrm{CH}_{2} \mathrm{O}-$ ), 4.36 (d.d, $1 \mathrm{H}, J=8.91,8.91, \mathrm{HO}_{-} \mathrm{CH}_{-}$), 4.50 (d.d, $1 \mathrm{H}$, $\left.J=8.91,8.91, \mathrm{HO}-\mathrm{C}_{2} \mathrm{I}^{-}\right), 4.58$ (d.d, $\left.1 \mathrm{H}, J=3.40,10.25, \mathrm{HO}_{-} \mathrm{CH}_{2}\right), 5.48$ (d, $1 \mathrm{H}, J=8.91$, -CH(OTBS)-Ar), 7.71-7.74 (m, 1H, Ar-H), 8.03-8.13 (m, 1H, Ar-H), 8.91-8.96 (m, 2H, $\mathrm{Ar}-\mathrm{H}),{ }^{13} \mathrm{C}-\mathrm{NMR}(100 \mathrm{MHz}, \mathrm{CDCl}) \mathrm{179.16}, 149.48,147.78,137.55,134.50,123.74,71.37$, $69.61,59.75,46.55,44.85,25.52,17.73,-4.07,-5.02$. Anal. Found: C, $61.28 ; \mathrm{H}, 8.00 ; \mathrm{N}$, 4.01. Calcd. for $\mathrm{C}_{17} \mathrm{H}_{2 i} \mathrm{NO}_{4} \mathrm{Si}: \mathrm{C}, 60.5 ; \mathrm{H}, 8.06 ; \mathrm{N}, 4.15 \%$.

4-Pyridyllactone (9c) Yield 79\%, white amorphous solid, mp $151-152^{\circ} \mathrm{C}$. ' $\mathrm{H}-\mathrm{NMR}$ $\left(400 \mathrm{MHz}, \mathrm{CDCl}_{s}, \mathrm{TMS}\right) \delta-0.42\left(\mathrm{~s}, 3 \mathrm{H},-\mathrm{Si}-\mathrm{CH}_{s}\right),-0.02\left(\mathrm{~s}, 3 \mathrm{H},-\mathrm{Si}_{-} \mathrm{CH}_{3}\right), 0.77(\mathrm{~s}, 9 \mathrm{H}$, $\left.-\mathrm{Si}-\mathrm{CH}_{i}\right), 2.67-2.64(\mathrm{~m}, 1 \mathrm{H},-\mathrm{CH}-\mathrm{CH}(\mathrm{OTBS})-\mathrm{Ar}), 2.93-2.95\left(\mathrm{~m}, 1 \mathrm{H}, \mathrm{HO}-\mathrm{CH}_{2}-\mathrm{CH}-\right), 3.55$ 5.43 (br.s, $1 \mathrm{H}, \mathrm{HO}-\mathrm{CH}_{2}-$ ) 3.68 (d.d, $1 \mathrm{H}, J=8.30,8.30$, $\mathrm{HO}-\mathrm{CH}_{2}-$ ), $3.89-4.01(\mathrm{~m}, 2 \mathrm{H}$, 
$-\mathrm{CH}_{\mathrm{O}} \mathrm{O}-$ ) 4.88 (d, 1H, $J=7.80,-\mathrm{CH}$ (OTBS)-Ar), 7.16-7.18 (m, 2H, Ar-H), 8.47-8.49 (m, $2 \mathrm{H}, \mathrm{Ar}-I)$ ) ${ }^{13} \mathrm{C}-\mathrm{NMR}(100 \mathrm{MHz}, \mathrm{CDCl})$ ) $178.83,151.10,150.08,121.60,121.35,72.81$, $69.74,59.94,46.20,44.80,25.63,17.88,-4.05,-4.50$. Anal. Found: C, 60.66; H, 8.00; N, 4.07. Calcd. for $\mathrm{C}_{17} \mathrm{H}_{27} \mathrm{NO}_{4} \mathrm{Si}: \mathrm{C}, 60.5 ; \mathrm{H}, 8.06 ; \mathrm{N}, 4.15 \%$.

4-11-(tert-Butyldimethylsilyl)oxy-1-pyridylmethyl]-3-methylenedihydro-2(3 H)-furanones (10)

To a solution of $9(240 \mathrm{mg}, 0.71 \mathrm{mmol})$ and triethylamine $(0.2 \mathrm{ml}, 1.42 \mathrm{mmol})$ in benzene $(50 \mathrm{ml})$ was added methanesulfonyl chloride $(0.1 \mathrm{ml}, 1.07 \mathrm{mmol})$ at $0^{\circ} \mathrm{C}$. After stirring for $3 \mathrm{hr}$ at room temperature, to the mixture was added saturated aqueous citric acid solution $(1 \mathrm{ml})$ and EtOAc $(50 \mathrm{ml})$. The organic layer was separated, washed with water, saturated aqueous $\mathrm{NaHCO}_{3}$ solution, brine, and dried $\left(\mathrm{Na}_{3} \mathrm{SO}_{4}\right)$. Concentration followed by column chromatography (silicagel, EtOAc) gave pure methylenelactones 10.

2-Pyridylmethylenelactone (10a) Yield 45\%, colorless amorphous solid, mp 68-69 ${ }^{\circ} \mathrm{C} .{ }^{\prime} \mathrm{H}-\mathrm{NMR}\left(400 \mathrm{MHz}, \mathrm{CDCl}_{3}, \mathrm{TMS}\right) \delta-0.27\left(\mathrm{~s}, 3 \mathrm{H},-\mathrm{Si}-\mathrm{CH}_{3}\right),-0.03\left(\mathrm{~s}, 3 \mathrm{H},-\mathrm{Si}-\mathrm{CH}_{3}\right), 0.80$ $\left(\mathrm{s}, 9 \mathrm{H},-\mathrm{Si}-\mathrm{CH}_{3}\right), 3.39-3.41(\mathrm{~m}, 1 \mathrm{H},-\mathrm{CH}-\mathrm{CH}(\mathrm{OH})-\mathrm{Ar}), 4.21-4.30\left(\mathrm{~m}, 2 \mathrm{H},-\mathrm{CH}_{2} \mathrm{O}-\right), 4.86$ (d, 1H, J=5.86, -CH(OTBS)-Ar), $4.87\left(\mathrm{~s}, 1 \mathrm{H}, \mathrm{CH}_{2}=\mathrm{C}-\right.$ ), 6.09 (s, $1 \mathrm{H}, \mathrm{CH}_{2}=\mathrm{C}-$ ), $7.12-7.15$ $(\mathrm{m}, 1 \mathrm{H}, \mathrm{Ar}-H), 7.29-7.31(\mathrm{~m}, 1 \mathrm{H}, \mathrm{Ar}-H), 7.60-7.64(\mathrm{~m}, 1 \mathrm{H}, \mathrm{Ar}-H), 8.45-8.46(\mathrm{~m}, 1 \mathrm{H}$, $\mathrm{Ar}-H) .{ }^{13} \mathrm{C}-\mathrm{NMR}\left(100 \mathrm{MHz}, \mathrm{CDCl}_{3}\right)$ 170.98, 160.64, 149.20, 136.56, 134.36, 124.36, 123.06, $121.84,77.40,68.56,45.78,25.92$, 18.18. Anal. Found: C, 63.91; H, 7.89; N, 4.05. Calcd. for $\mathrm{C}_{17} \mathrm{H}_{45} \mathrm{NO}$ Si: C, $63.91 ; \mathrm{H}, 7.89 ; \mathrm{N}, 4.38 \%$.

3-Pyridylmethylenelactone (10b) Yield 50\%: colorless oil. 'H-NMR (400 MHz, $\left.\mathrm{CDCl}_{3}, \mathrm{TMS}\right) \delta-0.28\left(\mathrm{~s}, 3 \mathrm{H},-\mathrm{Si}-\mathrm{CH}_{3}\right),-0.28\left(\mathrm{~s}, 3 \mathrm{H},-\mathrm{Si}-\mathrm{CH}_{3}\right), 0.80\left(\mathrm{~s}, 9 \mathrm{H},-\mathrm{Si}-\mathrm{CH}_{3}\right)$, $3.20-3.21(\mathrm{~m}, 1 \mathrm{H},-\mathrm{CH}-\mathrm{CH}(\mathrm{OTBS})-\mathrm{Ar}), 4.14-4.22\left(\mathrm{~m}, 2 \mathrm{H},-\mathrm{C} H_{2} \mathrm{O}-\right), 4.77(\mathrm{~d}, 1 \mathrm{H}, J=5.86$, $-\mathrm{CH}$ (OTBS)-Ar), $6.24\left(\mathrm{~s}, 1 \mathrm{H}, \mathrm{CH}_{2}=\mathrm{C}-\right), 6.25\left(\mathrm{~s}, 1 \mathrm{H}, \mathrm{CH}_{2}=\mathrm{C}-\right), 7.21-7.25(\mathrm{~m}, 1 \mathrm{H}, \mathrm{Ar}-H)$, $7.56-7.57(\mathrm{~m}, 1 \mathrm{H}, \mathrm{Ar}-H), 8.91-8.96(\mathrm{~m}, 2 \mathrm{H}, \mathrm{Ar}-H),{ }^{13} \mathrm{C}-\mathrm{NMR}\left(100 \mathrm{MHz}, \mathrm{CDCl}_{3}\right) 170.42$, $149.73,148.40,136.44,164.55,134.22,125.28,123.36,74.55,67.84,46.95,25.78,18.09$, $-4.36,-4.91$. Anal. Found: C, 61.37; H, 8.01; N, 4.44. Calcd. for $\mathrm{C}_{17} \mathrm{H}_{25} \mathrm{NO}_{3} \mathrm{Si}: \mathrm{C}, 63.91 ; \mathrm{H}$, $7.89 ; \mathrm{N}, 4.38 \%$.

4-Pyridylmethylenelactone (10c) Yield $88 \%$, white amorphous solid, mp $79-80^{\circ} \mathrm{C}$. 'H-NMR (400 MHz, $\left.\mathrm{CDCl}_{3}, \mathrm{TMS}\right) \delta-0.34\left(\mathrm{~s}, 3 \mathrm{H},-\mathrm{Si}-\mathrm{CH}_{3}\right), 0.03\left(\mathrm{~s}, 3 \mathrm{H},-\mathrm{Si}-\mathrm{CH}_{3}\right), 0.90(\mathrm{~s}$, $\left.9 \mathrm{H},-\mathrm{Si}-\mathrm{CH}_{3}\right), 3.15-3.16(\mathrm{~m}, 1 \mathrm{H},-\mathrm{CH}-\mathrm{CH}(\mathrm{OTBS})-\mathrm{Ar}), 4.15-4.24\left(\mathrm{~m}, 2 \mathrm{H},-\mathrm{CH}_{3} \mathrm{O}-\right) 4.70$ (d, $1 \mathrm{H}, J=5.40,-\mathrm{CH}$ (OTBS)-Ar), $5.21(\mathrm{~d}, 1 \mathrm{H}, j=0.98, \mathrm{CH}=\mathrm{C}-$ ), $6.23(\mathrm{~d}, 1 \mathrm{H}, j=1.46$, $\left.\mathrm{CH}_{2}=\mathrm{C}-\right), 7.14-7.15(\mathrm{ml}, 2 \mathrm{H}, \mathrm{Ar}-H), 8.53-8.54(\mathrm{~m}, 2 \mathrm{H}, \mathrm{Ar}-H),{ }^{19} \mathrm{C}-\mathrm{NMR}\left(100 \mathrm{MHz}, \mathrm{CDCl}_{3}\right)$ $170.26,149.94,149.71,133.72,125.29,121.63,75.46,67.76,46.53,25.64,18.01,1.02$, $-4.50,-4.32$. Anal. Found: $\mathrm{C}, 61.55 ; \mathrm{H}, 8.32 ; \mathrm{N}, 4.31$. Calcd. for $\mathrm{C}_{17} \mathrm{H}_{25} \mathrm{NO}_{3} \mathrm{Si}: \mathrm{C}, 63.91 ; \mathrm{H}$, $7.89 ; \mathrm{N}, 4.38 \%$.

\section{Lettuce seedling tests}

Lettuce (Lactuca sativa L. cv. Sacramento) seedling tests were performed by the same method as described previously (Kikuchi et al., 1990). The inhibitory activity of compounds was evaluated after 4 days by inspecting the rate of growth of the hypocotyls and roots. The inhibitory rates were determined by percentage of the averaged lengths of hypocotyls and roots of treated plants to those of controls and indicated according to the following scale: $\mathbf{0}<10 \% \leqq \mathbf{1}<30 \% \leqq \mathbf{2}<50 \% \leqq \mathbf{3}<70 \% \leqq \mathbf{4}<90 \% \leqq \mathbf{5}$ 


\section{RESULTS AND DISCUSSIONS}

\section{Synthesis}

The synthesis of ethyl 3-pyridyl-2-propenoates (1a-c) was accomplished by the reaction of 2-, 3-, and 4-pyridinecarboxaldehydes with diethyl ethoxycarbonylmethylphosphonate using potassium carbonate as a base. In the 2-pyridyl analog 1a, trans isomer 1a-1 produced with a small amount of cis isomer 1a-2 which was separated by column chromatography on silica gel. Confirmation of the structure of stereoisomers of 1a-1 and 1a-2 was provided from ${ }^{1} \mathrm{H}-\mathrm{NMR}$ spectra; the coupling constants between 2- $H$ and $3-H$ of $\mathbf{1 a - 1}$ and 1a-2 were observed as 15.9 and $12.7 \mathrm{~Hz}$, respectively. In the 3and 4-pyridyl analogs, cis isomers were not detected in the reaction mixture by TLC. The ethyl ester 1c was hydrolyzed to its corresponding carboxylic acid $\mathbf{2 c}$, which was treated with alkylamines in the presence of water-soluble carbodiimide and 1-hydroxybenzotriazole to give amides $\mathbf{3 c}$ (Scheme 1).

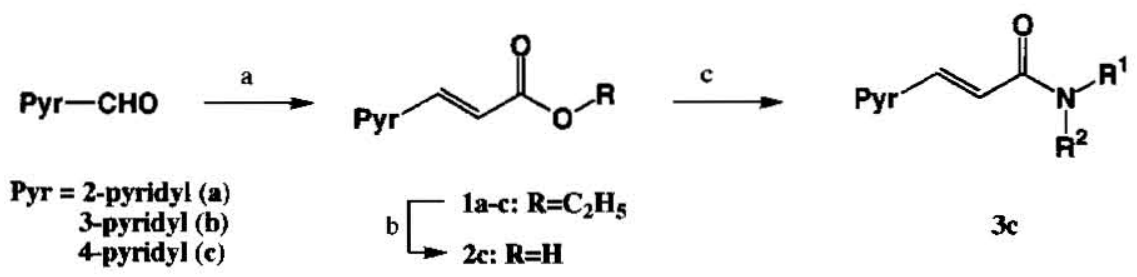

a) diethyl ethoxycarbonylmethylphosphonate, $\mathrm{K}_{2} \mathrm{CO}_{i}, \mathrm{H} \%$;

b) $\mathrm{NaOH}, \mathrm{EtOH}, \mathrm{H}_{2} \mathrm{O}$;

c) amine, 1-ethyl-3-(3-dimethylaminopropyl)carbodimide HCl, l-hydroxyberzotriazole, $\mathrm{CH}_{2} \mathrm{Cl}_{2}$

Scheme 1. Synthesis of pyridylpropenoic acid derivatives

The general synthetic pathway for the preparation of the pyridine derivatives possessing a $\gamma$-butyrolactone ring is shown in Scheme 2. $\beta$-Vinyl- $\gamma$-butyrolactone 4 was prepared according to the procedure reported (Kondo and Mori, 1974). The lithium enolate of 4 generated with 1.2 equivalent of lithium diisopropylamide (LDA) in tetrahydrofuran (THF) at $-78^{\circ} \mathrm{C}$ was treated with $2-, 3-$, and 4-pyridinecarboxaldehydes to give a mixture of crythro and threo aldol products in $87-90 \%$ yiclds in the respective ratio of c. a. 1:1, which was calculated based on their NMR spectra. Recrystallization from ethyl acetate afforded pure erythro 5a-c. After protecting a secondary hydroxyl group with $l$-butyldimethylsilyl chloride (TBSCl) in the presence of imidazole, the silyl ethers 6a-c were reduced with lithium aluminum hydride giving diols $\mathbf{7 a - c}$ quantitatively. Dihydroxylation of $\mathbf{7 a - c}$ by using a catalytic amount of osmium tetraoxide and 

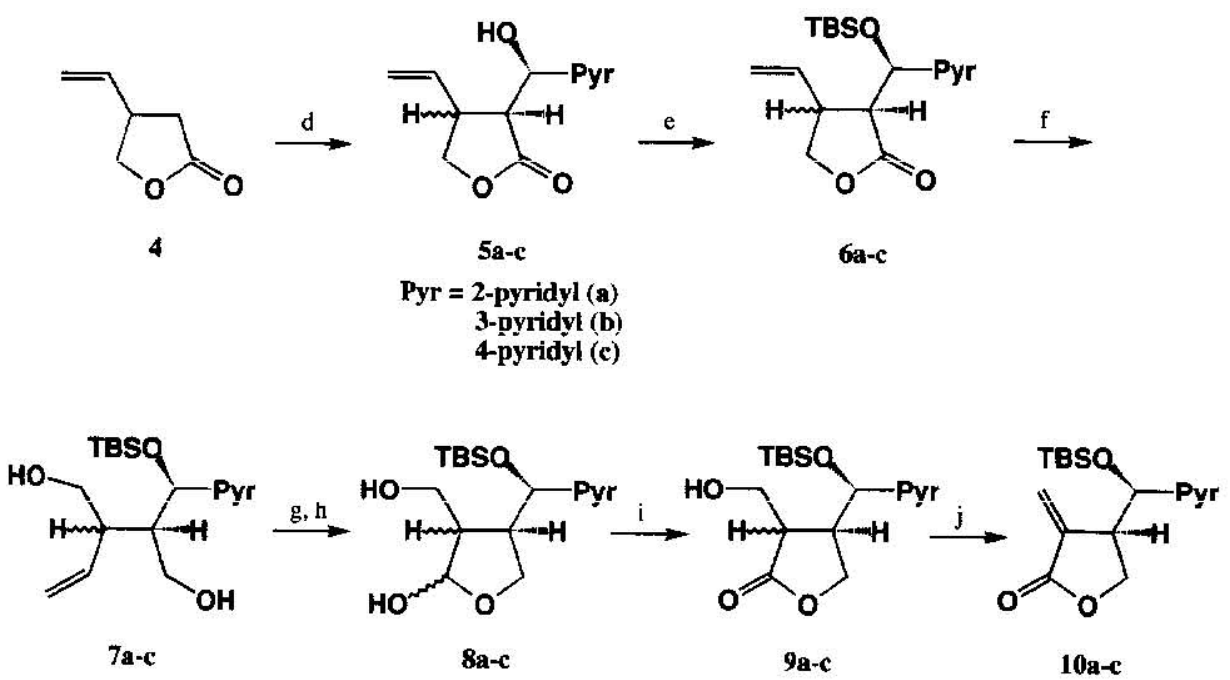

(1) LDA, Pyr-CHO, THF; e) TBSCl, imidazole, DMF; f) $\mathrm{L}_{j} \mathrm{AlH}_{2}, \mathrm{THF}$;

g) cat. $\mathrm{OsO}_{4}, \mathrm{NMO}$, acetone: $-\mathrm{BuOH}: \mathrm{H}, \mathrm{O}=4: 1: 1$; h) $\mathrm{NalO}_{3}$, aq. EtOAc

i) Ag CO-Celite, benzene; j) mesyl chloride, $\mathrm{Et}_{;} \mathrm{N}$, benzene.

Scheme 2. Synthesis of pyridine derivatives with a $\gamma$-butyrolactone ring

$N$-methylmorpholine $N$-oxide as a reoxidant and successive periodate oxidation afforded lactols $\mathbf{8 a - c . ~ T h e ~ l a c t o l s ~} \mathbf{8 a - c}$ were oxidized with silver carbonate-celite to lactones $\mathbf{9 a - c}$, which were treated with methanesulfonyl chloride in the presence of triethylamine to give $\mathbf{1 0 a - c . ~ T h e ~ m e s y l a t e d ~} \mathbf{9 a}-\mathbf{c}$ could not be isolated in this reaction, where the mesyl group eliminated without base to result in $\mathbf{1 0 a}-\mathbf{c}$.

\section{Pìant-growth inhibiting activity}

Table 1 shows the effects of a number of 3-pyridyl-2-propenoic acid derivatives on the growth of lettuce seedlings. The pyridine analogs of ethyl cinnamate (1a-1c) irrespective of the position of a nitrogen atom inhibited the growth of lettuce seedlings at $50 \mathrm{ppm}$. In the 2-pyridyl andogs, there was little difference in activity between cis and trans isomer. Among the ester analogs, the 4-pyridine isomer 1c showed the highest activity, which inhibited the growth of hypocotyl and root at $10 \mathrm{ppm}$. The activity of 3-(4-pyridyl) -2 -propenoic acid (2c) is less than that of the ester analog 1c. In contrast to the ester analogs, compound $\mathbf{2 c}$ had little effect on the hypocotyl growth. Since the 4-pyridinc analog showed considerably higher activity than other isomers, further modification was made in the compound 1c by replacing the ester group with an amide group. The $N$-propylamide $\mathbf{3 c - 1}$ had low inhibitory activity on comparison with the ester analog $\mathbf{1 c}$. The activity of amide analogs was found to fall off with increasing size of the $\mathrm{N}$-alkyl substituent. (3c-2), while the additional introduction of an alkyl group at the nitrogen 
atom (3c-3) ircreased the activity. Compound $\mathbf{3 c - 3}$ at $50 \mathrm{ppm}$ caused greater than $90 \%$ inhibition of the hypocotyl and root growth compared to the control, however, the activity rapidly decreased at $10 \mathrm{ppm}$. $N, N$-Diethyl cinnamamide at $50 \mathrm{ppm}$ had no activity on the growth of hypocotyl (data not shown), indicating that the presence of a nitrogen atom at the 4-position of the benzene ring was essential for the activity. Although it remains to be seen whether or not compounds $\mathbf{1 c}$ and $\mathbf{3 c - 3}$ inhibited cinnamate 4-hydroxylase in the lettuce seedlings, 3-(4-pyridyl)-2-propenoic acid derivatives might be a lead for the development of a new herbicide.

Table 1. Effects of pyridine derivatives on the growth of lettuce scedlings

\begin{tabular}{|c|c|c|c|c|c|c|}
\hline & \multirow{3}{*}{ Compound } & & \multicolumn{4}{|c|}{ Growth rate } \\
\hline & & & \multicolumn{2}{|c|}{ Hy[ocoly] } & \multicolumn{2}{|c|}{ Root } \\
\hline & & Conc. (ppm) & 50 & 10 & 50 & 10 \\
\hline 1a-1 & & & 2 & 0 & 2 & 0 \\
\hline 1a-2 & & & 2 & 0 & 2 & 1 \\
\hline $1 \mathrm{~b}$ & & & 2 & 1 & 2 & 0 \\
\hline lc & & & 3 & 2 & 4 & 3 \\
\hline $2 c$ & & & 1 & 0 & 3 & 2 \\
\hline $3 c-1$ & & & 3 & 2 & 3 & 1 \\
\hline $3 c-2$ & & & 2 & 1 & 1 & 1 \\
\hline $3 k-3$ & & & 5 & 2 & 5 & 2 \\
\hline
\end{tabular}

The bioassay data for pyridine derivatives with a $\gamma$-butyrolactone and related compounds on the growth of lettuce seedlings are given in Table 2 . In a series of pyridine analogs with a vinyllactone $(\mathbf{6 a - 6 c})$, the $3-$ and 4 -pyridine analogs $(\mathbf{6 b}$ and $\mathbf{6 c})$ at $10 \mathrm{ppm}$ 
inhibited the growth of hypocotyl and root more than $30 \%$ compared to the control, whereas the 2-pyridine analog $\mathbf{6 a}$ showed low activity even at $100 \mathrm{ppm}$. In contrast, all of the pyridine analogs with a $1,4-$ butanediol $(\mathbf{7 a - 7} \mathbf{c})$ and a methylenelactone $(\mathbf{1 0 a}-\mathbf{1 0} \mathbf{c})$ substituent had almost the same activity. It is noteworthy that the presence of the $\gamma-$ butyrolactone was dispensable for the activity.

The design of inhibitors targeted at the P450 enzymes which are involved in essential physiological functions in plants would be appear to be a promising field for future research. Further studies on this new series of pyridines are in progress.

Table 2. Elfects of pyridine derivatives with a $\gamma$-lactone ring and related compounds on the growth of lettuce seedlings

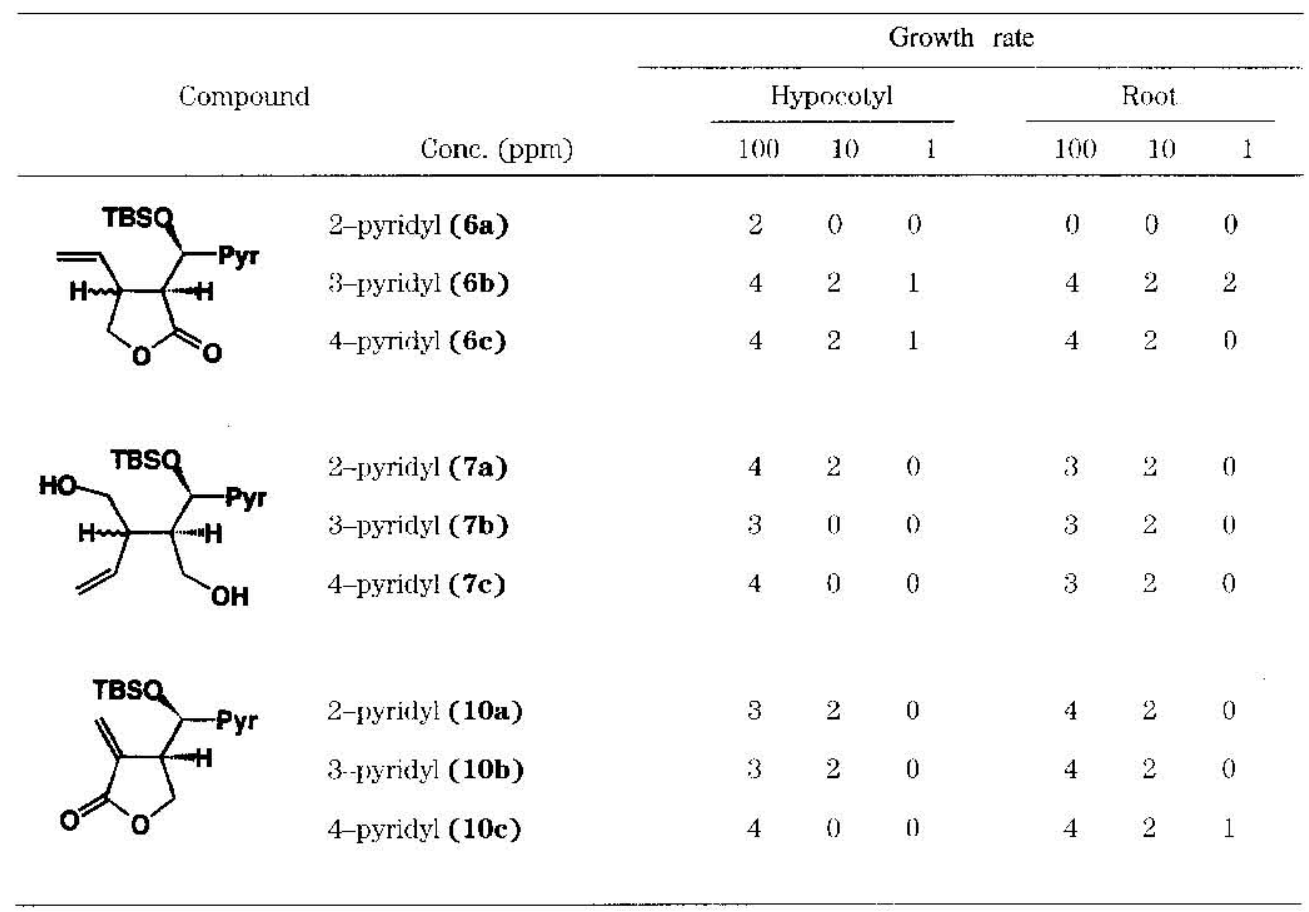

\section{REFERENCES}

Donaldson R. P. and D. G. Luster 1991 Multiple forms of plant cytochrome P-450. Plant Physiol., 96: $669-674$

Ishibashi F. and E. Taniguchi 1989 Syntheses of ( \pm -haedoxan A, D, E and their stereoisomers. Agric. Biol Chem., 53: $1565-1573$

Kikuchi M., E. Kuwano and M. Eto 1990 Synthesis and plant grow1h regulatory activity of 1,5-disubstituted ittidazoles. J. Fac. Agr., Kyushu Liniv., 34: 397-404 
Kondo K. and F. Mori 1974 Synthesis of $\gamma$-lactones by the condensation of 2-alkene-1,4-diols with orthocarboxylic esters. Chem. Lett., 741-742

Ortiz de Montellano, P. R. and N. O. Reich 1986 Inhibition of cytochrome P-450 enzymes. In "Cytochrome P-450", ed. by P. R. Ortiz de Montellano, Plenum Press, New York, pp. 273-314

Teutsch H. G., M. P. Hasenfratz, A. Lesot, C. Stoltz, J-M. Garner, J-M. Jeltsch, F. Durst and D. Werck-Reichhart 1993 Isolation and sequence of a cDNA encoding the Jerusalem artichoke cinnamate 4-hydrolase, a major plant cytochrome $\mathrm{P} 450$ involved in the general phenylpropanoid pathway. Proc. Natl. Acad. Sci. USA, 90: 4102-4106

Yamauchi S., F. Ishibashi and E. Taniguchi 1992 Insecticidal activity of sesquilignans with a 3-aryl-6-methoxy-2-methoxymethyl-1,4-benzodioxanyl group. Biosci. Biotech. Biochem., 56: $1760-1768$ 